\title{
Spiegeltherapie zur Behandlung chronischer Schmerzen
}

\author{
In den letzten Jahren hat die Spiegeltherapie, eine kognitive Therapie, zunehmend \\ Verbreitung gefunden. Sie bietet ein vielversprechendes Therapiepotenzial - auch \\ bei chronischen Schmerzen. Doch bevor sie in den Behandlungsplan aufgenommen \\ werden kann, sind ein paar Kriterien zu berücksichtigen. \\ Eduard Kraft
}

\section{Einleitung}

Die Spiegeltherapie wurde erstmals von Rahmanchandran 1996 beschrieben, der Patienten mit Phantomschmerz dieser Intervention unterzog und verblüffende akute Schmerzlinderung bei seinen Patienten erzeugen konnte [10]. Außer bei Schmerzerkrankungen wird die Spiegeltherapie in der Behandlung von motorischen Defiziten bei Patienten nach zerebraler Ischämie eingesetzt. Für diese Anwendung gibt es mittlerweile überzeugende Metaanalysen über die Wirksamkeit [12].

Die Behandlung chronischer Schmerzerkrankungen mit Spiegeltherapie wird im Wesentlichen bei der Therapie des Phantomschmerzes und beim komplex-regionalen Schmerzsyndrom (englische Abkürzung: CRPS) eingesetzt. Beides sind chronische Schmerzerkrankungen, die häufig schwer zu behandeln sind und den Patienten in seiner Funktionsfähigkeit und Teilhabe erheblich und zum Teil über Jahre einschränken. Daher eröffnen innovative Ansätze wie die Spiegeltherapie wertvolle therapeutische Optionen.

Im Gegensatz zur Behandlung motorischer Schwäche nach Schlaganfall ist die Datenlage der bisher stattgefundenen klinischen Studien schwieriger zu interpretieren $[6,4,9]$.

\section{Praktische Durchführung der Spiegeltherapie \\ Einzeltherapie}

Die meisten Erfahrungen liegen im Setting der Einzeltherapie bei Spiegeltherapie vor. Hierzu wird der Patient so an den Spiegel gesetzt, dass die betroffene Extremität verdeckt ist. Der Patient blickt dabei auf das Abbild seines gesunden Armes oder Beines. Dies führt zu der optischen Illusion, die bei Phantomschmerz suggeriert, die amputierte Extremität sei vorhanden. Im Fall des CRPS erscheint die betroffene Extremität nicht verändert zu sein.
In der Literatur und den verschiedenen Einrichtungen, die betroffene Patienten behandeln, werden unterschiedliche Protokolle und Modifikationen der Spiegeltherapie verwendet.

Wichtige Hinweise zur Durchführung Bevor jedoch die Spiegeltherapie im Behandlungsplan eingesetzt werden sollte, empfiehlt es sich, einige Kriterien bei der Durchführung und Planung zu berücksichtigen:

- Wichtig ist in diesem Zusammenhang, auf den aktuellen Patientenzustand einzugehen und den Patienten vorsichtig an die geplante Intervention heranzuführen. Dabei sollte es vermieden werden, den Patienten kognitiv oder emotional zu überfordern.

- Psychisch instabile Patienten sind häufig nicht für die Anwendung der Spiegeltherapie geeignet.

- Eine ausreichende kognitive Belastbarkeit für Konzentrationsfähigkeit und Aufmerksamkeit sollte vorab beurteilt werden.

- Auch muss berücksichtigt werden, dass häufig auch die gesunden Extremitäten pathologische Veränderungen aufweisen können (z.B. Dupuytren-Kontrakturen an der Hand). Dies würde die Effektivität der Spiegeltherapie beeinträchtigen.

- Der Therapieraum sollte so gestaltet sein, dass möglichst reizarme Raumbedingungen angeboten werden können. So sind viele visuelle Ablenkungsphänomene, wie andere sich im Raum befindliche Personen oder offene Fenster und Mobiliar, kritisch zu prüfen.

- Es empfiehlt sich, Spiegelkonstruktionen zu verwenden, die ausreichend verhindern, dass die betroffene Extremität hinter dem Spiegel sichtbar ist. Insbesondere bei unteren Extremitäten muss daher häufig auf einen Standspiegel zurückgegriffen werden.
- Häufig sind praktische Aspekte, z.B. wie stand- und bruchsicher die Spiegelkonstruktion ist, ebenso zu berücksichtigen.

- Auch können höhenverstellbare Tische und Rückenlehnen erhebliche Erleichterung für den Patienten im Training darstellen.

Dauer der Interventionseinheiten Ungeachtet der unterschiedlichen publizierten Protokolle wie Graded Motor Imagery, dem Protokoll aus Bath (England), oder dem Bonner, Berliner und St. Gallener Protokoll, gibt es relevante Gemeinsamkeiten. Alle diese Protokolle verwenden kurze Interventionseinheiten von wenigen Minuten.

\section{Ablauf in unserer Klinik}

In unserer Klinik wird bei der Anwendung der Spiegeltherapie die Extremität zunächst ohne Bewegung mit kleinen Pausen hintereinander vom Patienten im Spiegel betrachtet. Dieser Abschnitt sollte auf bis zu $10 \mathrm{Mi}$ nuten begrenzt sein.

Dann werden die Bewegungsabläufe der Extremität mit steigender Komplexität durchgeführt. Auch diese Phase sollte maximal 10 Minuten anhalten.

Im weiteren Verlauf kann eine letzte Phase mit Einsatz von Therapiematerialien ergänzt werden. Sensorisches Therapiematerial, wie der Igelball ( $\odot$ Abb. 1), bieten sich hier an. Verschiedene Arten von sensorischer Stimulation werden von den Patienten häufig als angenehm und entspannend wahrgenommen.

Alle Patienten sollten im Verlauf ihre Therapieeinheiten durch Heimübungen ergänzen.

\section{Spiegeltherapie in der Gruppe}

Vor Kurzem haben Mückel und Mehrholz die Anwendung der Spiegeltherapie in der Gruppe diskutiert [8]. Gemäß den Autoren kann sie eine effiziente Alternative zur Einzeltherapie darstellen. Eine kürzlich erschienene Studie mit einer Spiegeltherapiegruppe konnte ebenso wie die Einzeltherapie eine deutliche Steigerung der Motorik bei den untersuchten Patienten nachweisen [11]. Allerdings muss berücksichtigt werden, dass erhöhte Anforderungen an die Konzentrationsfähigkeit gestellt werden und daher eine Gruppengröße von mehr als vier Patienten nicht für sinnvoll erachtet wird. Auch die Anordnung der Therapieplätze ist etwas aufwendig, da größere Abstände eingehalten werden müssen. 


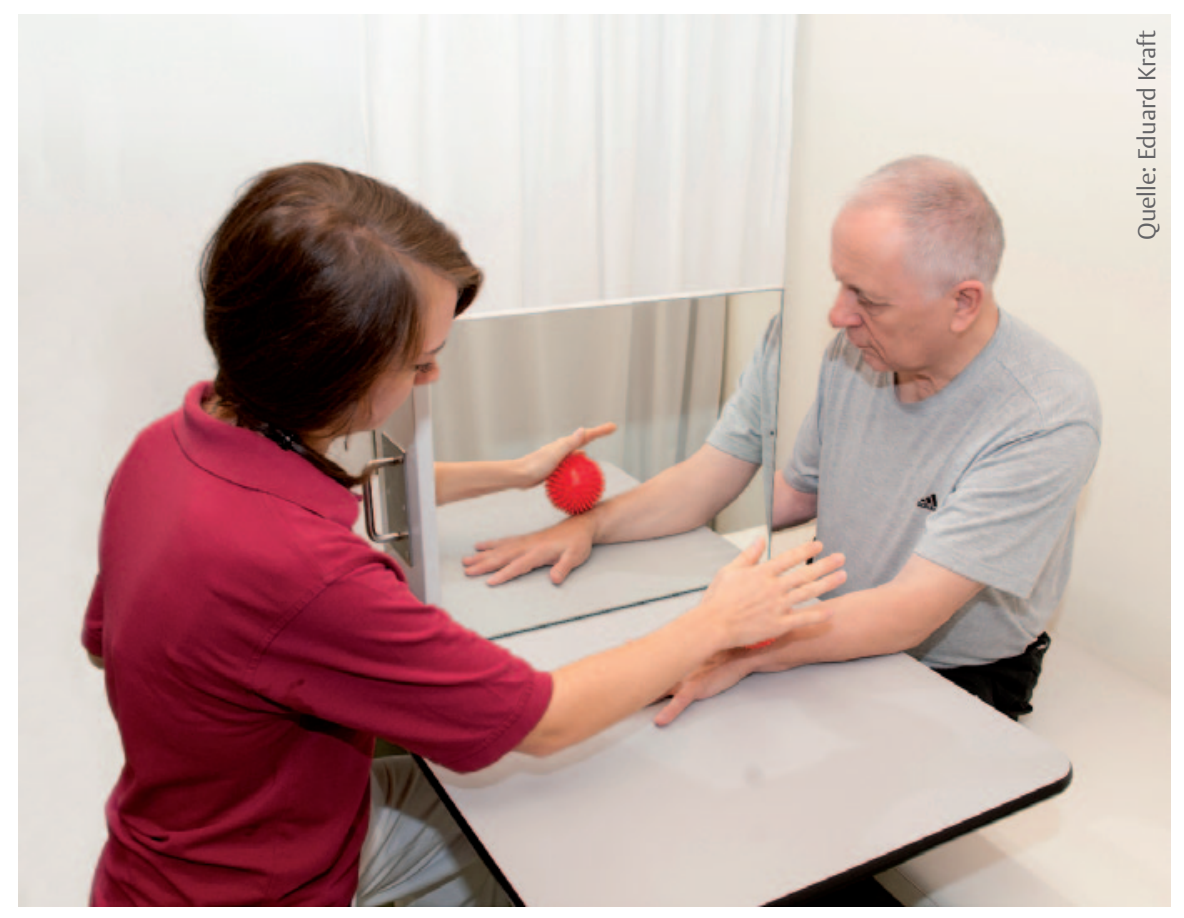

Abb. 1 Die Anwendung der Spiegeltherapie bei einem Patienten mit sensorischer Stimulation (Igelball).

Nicht alle Patienten profitieren von der Spiegeltherapie. Die aktuellen Daten deuten darauf hin, dass eine erfolgreiche Behandlung damit in Zusammenhang steht, ob die Patienten in der Lage waren, die gespiegelten Bewegungen zu ihren Phantomextremitäten in Verbindung zu setzen. Wenngleich alle Patienten im Schnitt eine Erhöhung dieser Fähigkeit während der Behandlung beschrieben, war die Ausgangssituation doch sehr unterschiedlich. Das liegt nahe, da die Fähigkeit, sich in ein Spiegelbild hineinzudenken, eine Grundvoraussetzung des Patienten ist, die er in die Therapie einbringt.

\section{Hypothesen zur Wirkung der Spiegeltherapie}

Eine der neurobiologischen Hypothesen zur Entstehung chronischer Schmerzen ist die sogenannte maladaptive kortikale Reorganisation. Allerdings ist der genaue Mechanismus noch sehr umstritten (siehe Beitrag zu den Grundlagen chronischer Schmerzen in diesem Heft [5]).

Veränderungen des Körperschemas So können sich zum Beispiel Patienten mit Phantomschmerz, die ein zusätzlich ausgeprägtes Teleskoping (Verkürzung der Phantomhand) aufweisen, nicht in die Spiegeltherapie hineinversetzen, weil sie vielleicht ihre ver- kürzt repräsentierte Hand nicht mit der im Spiegel sichtbaren Hand zur Deckung bringen können [3].

Vor Kurzem wurde das Konzept der Körpermatrix (Body Matrix) vorgeschlagen, das die Fähigkeit beschreibt, bestimmte Extremitäten, die nicht zum eigenen Körper gehören, wie z. B. bei der Rubber Hand Illusion, in das Körperschema einbeziehen zu können [7]. Dieses sogenannte Embodiment ist bei Gesunden sehr unterschiedlich verbreitet, wie Untersuchungen von Berkrater-Bodmann und Kollegen belegen konnten [1].

\section{Zusätzlich konnte auch gezeigt} werden, dass die Einbeziehung eines fremden Gegenstands in das eigene Körperschema und nicht die Bewegung des Phantoms notwendig ist, um Schmerz zu reduzieren. Die Fähigkeit, die gespiegelte Hand tatsächlich in das Körperschema einzubeziehen, konnte voraussagen, ob es zu einer Schmerzverbesserung bei einem Patienten mit Phantomschmerz kommen würde.

Veränderungen der zerebralen Repräsentation In diesem Zusammenhang ist der inferiore parietale Kortex eine interessan- te Region, die möglicherweise sowohl die Modifikation des aktiven Körperschemas als auch die schmerzhaften sensorischen Wahrnehmungen integriert. Damit dieser Mechanismus funktioniert, muss der Patient die gespiegelte Hand akzeptieren. Allerdings existieren erhebliche individuelle Unterschiede, wie der Patient in der Lage ist, sich in die gespiegelte Extremität einzudenken.

Dies könnte ein Grund dafür sein, dass Patienten mit Teleskoping überhaupt keinen Nutzen von der Spiegeltherapie hatten. Hingegen beschrieben Patienten ohne Teleskoping-Phänomen eine durchschnittliche Schmerzreduktion von über 50\% [3].

Deconinck und Mitarbeiter haben in einer gerade vorgestellten Literaturübersicht den Effekt der Spiegeltherapie bei motorischen Störungen nach zerebraler Ischämie zusammenfassend dargestellt [2]. Basierend auf 33 Studien konnten diese Autoren zeigen, dass die Regionen, die perzeptuelle, motorische und höhere kognitive Funktionen repräsentieren, die Areale im Gehirn sind, die durch die Spiegeltherapie beeinflusst werden. Entsprechend dieser Arbeiten werden insbesondere drei zerebrale Netzwerke durch die Spiegeltherapie beeinflusst:

- Das erste Netzwerk ist für die Verarbeitung von Aufmerksamkeitsprozessen verantwortlich. Es schließt Bereiche des Parietalkortex, visuelle Regionen im Okzipitalkortex sowie somatosensorische Areale, den cingulären Kortex und die Inselregion ein.

- Ein weiteres Netzwerk besteht aus Bereichen im temporalen und prämotorischen Kortex. Dabei scheinen Anteile des Spiegelneuronensystems miterfasst zu werden. Spiegelneurone sind für die Imitation und das Erkennen der Bedeutung von Bewegungen und für motorisches Lernen relevant.

- Das dritte zerebrale Netzwerk ist das motorische Netzwerk und im Besonderen der ipsilaterale primäre motorische Kortex (auf der Seite der betroffenen Extremität). Möglicherweise kann die Erregungsschwelle der Neurone im motorischen Kortex durch die Spiegeltherapie verändert werden. Dadurch kann der mögliche Funktionsschaden, der durch einen erlernten Nichtgebrauch verursacht werden kann, deutlich schwächer ausfallen. 
Bezogen auf die positiven Effekte der motorischen Beeinträchtigung würde die Spiegeltherapie Effekte generieren, die ähnlich den Mechanismen sind, denen motorisches Lernen zugrunde liegt. Dabei handelt es sich um die positiven Wirkungen der Wahrnehmungsfokussierung und kognitiven Verarbeitung von motorischen Handlungen.

Zusätzlich scheint die motorische Funktion durch eine Bahnung des primär motorischen Kortex in der betroffenen Hemisphäre verbessert zu werden. Auch zeigen die bisherigen Bildgebungsstudien, dass ein klarer Unterschied zwischen der Beobachtungstherapie, der Bewegungsvorstellung und der Spiegeltherapie besteht, da nicht alle das gleiche Muster des motorischen Netzwerkes abbilden.

\section{Ausblick}

Zweifellos werden weitere ausreichend groß angelegte Studien benötigt werden, um die Wirksamkeit der Spiegeltherapie zu untermauern. Derzeit gibt es nach den Kriterien der evidenzbasierten Medizin keinen eindeutigen Wirkungsnachweis bei den beschriebenen chronischen Schmerzerkrankungen [9]. Dennoch ist die Spiegeltherapie gemäß unseres aktuellen Verständnisses des Schmerzes biologisch und klinisch überaus plausibel.

Autor
$\begin{aligned} & \text { Eduard Kraft ist Leiter der } \\ & \text { interdisziplinären Schmerz- } \\ & \text { ambulanz am Klinikum der } \\ & \text { Ludwig-Maximilians-Universität } \\ & \text { in München. }\end{aligned}$
$\begin{aligned} & \text { Dr. med. Eduard Kraft } \\ & \text { Geschäftsführender Ober- } \\ & \text { arzt der Klinik für Orthopädie, }\end{aligned}$
Physikalische Medizin und Rehabilitation, Klinikum
Marchioninistr.15
81377 München
E-Mail: eduard.kraft@med.uni-muenchen.de

\section{Literatur}

1. Bekrater-Bodmann R, Foell J, Diers M, Flor H. The perceptual and neuronal stability of the rubber hand il lusion across contexts and over time. Brain Research 2012; 3(1452): 130-139. PubMed PMID: 22459041

2. Deconinck FJ, Smorenburg AR, Benham A et al. Reflections on mirror therapy: A systematic review of the effect of mirror visual feedback on the brain. Neurorehabilitation and Neural Repair 2014; 26. PubMed PMID: 25160567

3. Foell J, Bekrater-Bodmann R, Diers M, Flor H. Mirror therapy for phantom limb pain: Brain changes and the role of body representation. European Journal of Pain 2014; 18(5):729-739. PubMed PMID: 24327313

4. Johnson S, Hall J, Barnett $S$ et al. Using graded motor imagery for complex regional pain syndrome in clinical practice: Failure to improve pain. European Journal of Pain 2012; 16(4): 550-561. PubMed PMID: 22337591

5. Lotze M. Neurobiologie des Schmerzes. neuroreha 2015; 7(1): 9-15

6. Moseley GL. Graded motor imagery for pathologic pain: A randomized controlled trial. Neurology 2006; 67(12): 2129-2134. PubMed PMID: 17082465

7. Moseley GL, Gallace A, Spence C. Bodily illusions in health and disease: physiological and clinical perspectives and the concept of a cortical „body matrix". Neuroscience and Biobehavioral Reviews 2012; 36(1): 34-46. PubMed PMID: 21477616

8. Mückel S, Mehrholz J. Indikationen für die Spiegeltherapiegruppe. neuroreha 2014; 6: 78-79

9. O'Connell NE, Wand BM, McAuley J, Marston L, Moseley GL. Interventions for treating pain and disability in adults with complex regional pain syndrome. The Cochrane Database of Systematic Reviews 2013; 4: CD009416. PubMed PMID: 23633371

10. Ramachandran VS, Rogers-Ramachandran D. Synaesthesia in phantom limbs induced with mirrors. Proceedings Biological Sciences/The Royal Society 1996; 263(1369): 377-386. PubMed PMID: 8637922

11. Thieme H, Bayn M, Wurg M et al. Mirror therapy for patients with severe arm paresis after stroke - a randomized controlled trial. Clin Rehabil 2013; 4: 314--324

12. Thieme $\mathrm{H}$, Mehrholz J, Pohl M, Behrens ], Dohle $\mathrm{C}$. Mirror therapy for improving motor function after stroke. Stroke 2013; 44(1): e1-2. PubMed PMID: 23390640

Bibliografie

DOI $10.1055 / \mathrm{s}-0035-1548528$

neuroreha 2015; 7: 37-39

(c) Georg Thieme Verlag KG

Stuttgart · New York · ISSN 1611-6496 